\title{
Evaluation of the validity and utility of a transdiagnostic psychosis dimension encompassing schizophrenia and bipolar disorder
}

Ulrich Reininghaus, * Jan R. Böhnke, * Georgina Hosang, Anne Farmer, Tom Burns, Peter McGuffin and Richard P. Bentall

\section{Background}

In recent years, the Kraepelinian dichotomy has been challenged in light of evidence on shared genetic and environmental factors for schizophrenia and bipolar disorder but empirical efforts to identify a transdiagnostic phenotype of psychosis remain remarkably limited.

\section{Aims}

To investigate whether schizophrenia spectrum and bipolar disorder lie on a transdiagnostic spectrum with overlapping non-affective and affective psychotic symptoms.

\section{Method}

Multidimensional item-response modelling was conducted on symptom ratings of the OPerational CRITeria (OPCRIT) system in 1168 patients with schizophrenia spectrum and bipolar disorder.

\section{Results}

A bifactor model with one general, transdiagnostic psychosis dimension underlying affective and non-affective psychotic symptoms and five specific dimensions of positive, negative, disorganised, manic and depressive symptoms provided the best model fit and diagnostic utility for categorical classification.

\section{Conclusions}

Our findings provide support for including dimensiona approaches into classification systems and a directly measurable clinical phenotype for cross-disorder investigations into shared genetic and environmental factors of psychosis

\section{Declaration of interest}

None.

\section{Copyright and usage}

(c) The Royal college of Psychiatrists 2016.
The dichotomy of dementia praecox/schizophrenia and manic depression has informed diagnostic classification in psychiatry ever since it was first proposed by Emil Kraepelin. ${ }^{1}$ Although this dichotomy remains in DSM $-5,{ }^{2}$ the need for a dimensional approach to classification in psychiatry, ${ }^{3}$ and inclusion of such an approach in updated versions of DSM $-5^{4}$ and the impending ICD- $11^{5}$ continues to be debated in view of calls for research that looks across diagnostic categories ${ }^{3,5}$ and the persistent challenge of high comorbidity rates. ${ }^{3-8}$ In recent years, the Kraepelinian dichotomy has been challenged in light of evidence on common aetiological factors in schizophrenia and bipolar disorder. ${ }^{9}$ Evidence has accumulated that genetic risk is partly shared between schizophrenia and bipolar disorder. ${ }^{9-12}$ For instance, molecular genetic studies point to common DNA variants that have an impact on the risk of both disorders. ${ }^{11}$ There is also evidence of shared environmental contributions to schizophrenia and bipolar disorder. ${ }^{12}$ These include prenatal factors, ${ }^{13,14}$ childhood adversity, ${ }^{15}$ substance misuse ${ }^{16,17}$ urbanicity $^{18,19}$ and ethnicity. ${ }^{19}$ These findings, taken together, challenge Kraepelin's ${ }^{1}$ distinction between schizophrenia and bipolar disorder and their classification as separate diagnostic entities. Alternatively, Craddock \& Owen ${ }^{9}$ have proposed a transdiagnostic, mood-psychosis clinical dimension with three overlapping broad domains of psychopathology, namely schizophrenia, schizoaffective disorder and bipolar disorder. However, there is also evidence of genetic and environmental risks that are not shared between schizophrenia and bipolar disorder. Family studies have consistently reported unique genetic contributions to schizophrenia and bipolar disorder. ${ }^{12}$ Findings further suggest some degree of specificity of environmental exposures. ${ }^{8,18,19}$

*These authors are joint first authors.
Although, coupled with evidence on differences in course and outcome, ${ }^{8}$ these findings support the heterogeneity of psychotic disorders, it remains unclear which general and/or specific domains of the clinical psychosis phenotype will be most useful to measure and map onto genetic and environmental factors and their underlying biological and psychological mechanisms. ${ }^{9}$

Intriguingly, empirical efforts to identify a more fundamental, transdiagnostic phenotype of psychosis at the clinical symptom level remain remarkably limited. Whereas previous factor-analytic work has largely pointed towards a pentagonal model with five dimensions of positive symptoms, negative symptoms, cognitive disorganisation, mania and depression, ${ }^{20}$ we have reported evidence for a bifactor model that includes one general psychosis dimension underlying affective and non-affective psychotic symptoms as well as five specific psychosis dimensions of positive symptoms, negative symptoms, disorganisation, mania and depression. ${ }^{21}$ However, findings were restricted to samples of schizophrenia spectrum disorder and the clinical symptom measure (i.e. the Positive and Negative Syndrome Scale) not directly linked to existing diagnostic classification systems. Also, in contrast to our finding, Russo et al ${ }^{22}$ reported a model with two distinct factors for non-affective and affective psychosis. Empirical evidence on general and/or specific symptom dimensions, and their diagnostic utility, in both schizophrenia spectrum and bipolar disorder may help psychiatry move towards diagnostic approaches that better match shared and non-shared genetic and environmental risks, on the basis of which treatment and prognosis can be optimised. In this study, we aimed to investigate whether schizophrenia spectrum and bipolar disorders lie on a transdiagnostic spectrum with overlapping non-affective and affective psychotic symptoms using a symptom measure that can be directly used for making clinical diagnosis, i.e. the 
OPerational CRITeria (OPCRIT) system. ${ }^{23}$ To this end, we sought to examine: (a) whether the previously identified general psychosis dimension holds across diagnostic categories of schizophrenia spectrum and bipolar disorders; (b) whether formation of specific psychosis dimensions is justified in addition to a general psychosis dimension; (c) the diagnostic utility of general and specific psychosis dimensions for classifying patients correctly into categorical diagnoses of psychotic disorders; and (d) associations between clinical variables and general and specific psychosis dimensions.

\section{Method}

\section{Sample}

We analysed a pooled sample obtained from the UK700 study ${ }^{24}$ and the Bipolar Association Case-Control Study (BACCS). ${ }^{25}$ Patients in the UK700 study were recruited between 1994 and 1996 from four UK inner-city mental health services in London and Manchester using the following inclusion criteria: (a) aged between 18 and 65 years; (b) a psychotic illness for at least 2 years. A total of 708 patients were recruited during the study period. Of these, 691 patients with a diagnosis of schizophrenia $(n=345)$, schizoaffective disorder $(n=270)$, mania/bipolar disorder $(n=34)$ and unspecified functional psychoses $(n=42)$ were included in the current study. Patients with a diagnosis of major depression $(n=16)$ or without diagnosis $(n=1)$ were excluded.

Patients in the BACCS study ${ }^{25}$ were recruited between 2004 and 2007 from the greater London area, UK, through out-patient psychiatric clinics, self-help groups and media advertisements. Inclusion criteria were: (a) aged over 18 years; (b) at least two episodes of illness, at least one of which fulfilled diagnostic criteria for mania/hypomania. During the study period, a total of 512 patients were recruited at the London site. Of these, 477 patients with a current diagnosis of mania/bipolar disorder $(n=332)$, hypomania $(n=143)$ and unspecified functional psychosis $(n=2)$ were included in the current study. Patients with a current diagnosis of major depression $(n=2)$ and current unspecified diagnosis $(n=33)$ were excluded. More detailed information including ethical approval for all relevant aspects of the studies is available in Burns et $a l^{24}$ and Cohen-Woods et al. ${ }^{25}$ The two samples were combined in a pooled sample to achieve both sufficient numbers in each diagnostic group and a sufficient prevalence of individual symptoms for item-response model analysis to be performed.

\section{Measures}

The OPCRIT system was used to assess psychiatric symptoms as described by McGuffin et al. ${ }^{23}$ OPCRIT consists of a 90-item checklist that allows for structured examination of basic demographic information, disease course and severity (including age at onset, mode of onset, premorbid adjustment), and psychotic symptoms based on all available sources including case records, clinical and research interviews. It provides definitions for each item and algorithms for objective diagnosis of non-affective and affective psychotic disorders based on a range of diagnostic classification systems. A detailed description of the use of OPCRIT in the BACCS and UK700 study is provided in online supplement DS1. For the purposes of this study, psychiatric diagnosis was made based on the Research Diagnostic Criteria (RDC) using the OPCRIT system, ${ }^{26}$ which has been found to be both feasible and reliable in research settings and has been redesigned for use in clinical settings (i.e. OPCRIT+). ${ }^{23,27}$ We used all OPCRIT items with sufficient prevalence of psychotic symptoms $(>10 \%)$ in the pooled sample for item-response model analysis.

\section{Statistical analysis}

Multidimensional item-response modelling was conducted using the mirt package ${ }^{28}$ of the $\mathrm{R}$ environment ${ }^{29}$ for model estimation. We assumed data to be missing at random, which allowed for inclusion of the full sample. We examined model fit using the log-likelihood, the Akaike information criterion (AIC), Bayesian information criterion (BIC) and the sample-size adjusted BIC (SABIC). ${ }^{30}$ For these fit statistics, lower values than for the comparison model indicate a statistically better model fit. To examine whether there is a general psychosis dimension and, in addition, whether there are five specific symptom dimensions (positive symptoms, negative symptoms, disorganisation, mania, depression), we estimated five alternative item-response models (online Fig. DS1): first, a simple unidimensional model with one general factor explaining all OPCRIT symptom ratings (model A; corresponding to a unitary psychosis model); second, a multidimensional model with five uncorrelated specific factors for each specific symptom dimension (model B); third, a multidimensional model with five correlated specific factors (model C; corresponding, as model $\mathrm{B}$, to the pentagonal model of psychosis $^{20}$ ); fourth, a bifactor model with two distinct factors for affective and nonaffective psychosis and five uncorrelated factors for each specific symptom dimension (model D; corresponding to the Russo et al $^{22}$ model); and, fifth, a bifactor model with one general factor independent from five uncorrelated (orthogonal) specific factors (model E; corresponding to the bifactor model reported in our earlier study of patients with schizophrenia spectrum disorder ${ }^{21}$ ). The procedure for fitting these models in the context of psychotic symptom ratings have been described in more detail elsewhere. ${ }^{21}$ Importantly, the bifactor model with one general and five specific factors constrained each OPCRIT item to have a non-zero loading on the general factor (i.e. psychosis) and a non-zero loading on a specific factor (for example positive symptoms) to examine whether there is a general dimension underlying symptoms of schizophrenia spectrum and bipolar disorder independent from (i.e. non-redundant with) the previously reported specific symptom dimensions. To examine the extent to which factor scores of general and specific psychosis dimensions (as predictor variable) allow for accurate classification of patients into diagnostic categories (as outcome variable), multinomial receiver operating characteristic (ROC) analysis ${ }^{31}$ were conducted in Stata version 12. Finally, we used linear regression to investigate associations between clinical variables (including age at onset, mode of onset, premorbid work adjustment and premorbid social adjustment) and factor scores of general and specific psychosis dimensions.

\section{Results}

\section{Basic sample characteristics}

Basic sample characteristics of the pooled sample of 1168 patients are summarised in online Table DS1. The mean age at interview was 42.1 years and approximately half were female $(n=608$, $52.1 \%)$. The mean age at illness onset was 22.2 years. The most common diagnosis was mania/bipolar disorder (31.3\%), followed by schizoaffective disorder (29.5\%) and schizophrenia (23.1\%). As can be seen in online Table DS2, the prevalence of psychotic symptoms was sufficient in the pooled sample for item-response model analysis. Consistent with inclusion criteria, we observed differences in prevalence of symptoms across studies. 


\begin{tabular}{|c|c|c|c|c|c|}
\hline & \multicolumn{5}{|c|}{ Full information fit statistics ${ }^{a}$} \\
\hline & $\mathrm{LL}$ & FP & AIC & $\mathrm{BIC}$ & SABIC \\
\hline Unidimensional (unitary) model (A) & -24624.19 & 98 & 49444.39 & 49940.57 & 49629.29 \\
\hline Multidimensional (pentagonal) model with uncorrelated factors (B) & -23585.87 & 98 & 47367.74 & 47863.92 & 47552.64 \\
\hline Multidimensional (pentagonal) model with correlated factors (C) & -22468.5 & 108 & 45153.00 & 45175.23 & 45356.76 \\
\hline $\begin{array}{l}\text { Bifactor model with } 2 \text { factors for affective and non-affective psychosis } \\
\text { and } 5 \text { specific symptom factors (D) }\end{array}$ & -22539.24 & 147 & 45372.49 & 46116.75 & 45415.14 \\
\hline Bifactor model with 1 general psychosis and 5 specific symptom factors $(E)^{b}$ & -22058.87 & 147 & 44411.75 & 45156.02 & 44689.09 \\
\hline \multicolumn{6}{|c|}{$\begin{array}{l}\text { LL, log-likelihood; FP, free parameters; AIC, Akaike information criterion; BIC, Bayesian information criterion; SABIC, sample-size adjusted Bayesian information criterion. } \\
\text { a. A difference of } 10 \text { in AIC, BIC and SABIC is considered important. } \\
\text { b. The best model fit was still observed for model } E \text { as compared with a bifactor model, in which factor loadings of mania items on the general factor were fixed to zero and the } \\
\text { specific mania factor correlated with the general and other specific factors }\left(\mathrm{LL}=-22104.99, \mathrm{AIC}=44491.98, \mathrm{BIC}=45205.87, \mathrm{SABIC}=44758.01 ; r_{\text {general-mania }}=-0.68, r_{\text {positive-mania }}=-0.14\right. \\
\left.r_{\text {negative-mania }}=-0.27 ; r_{\text {disorganisation-mania }}=0.21 ; r_{\text {mania-depression }}=0.15\right) \text {. }\end{array}$} \\
\hline
\end{tabular}

\section{Dimensionality of schizophrenia spectrum and bipolar disorder}

Model fit statistics for the five alternative dimensional models of schizophrenia spectrum and bipolar disorder are shown in Table 1 . The best model fit was consistently observed across model fit statistics for the bifactor model including one general and five specific symptom factors compared with all other models. This indicated that there was a general psychosis dimension that explained associations among all symptoms of schizophrenia spectrum and bipolar disorder as well as that, over and above this general psychosis dimension, the formation of five specific psychosis dimensions was justified.

\section{Association of symptom ratings with general and specific psychosis dimensions}

Online Table DS3 shows standardised factor loadings for the best-fitting model including one general and five specific psychosis dimensions. Factor loadings on the general psychosis factor were moderate to strong for most OPCRIT symptom ratings. OPCRIT ratings of manic symptoms were inversely related to the underlying general psychosis dimension. By contrast, factor loadings of almost all other ratings were in the positive range. Coupled with findings on model fit statistics reported above, this indicated that there is a general psychosis dimension underlying affective and non-affective psychotic symptoms that holds across schizophrenia spectrum and bipolar disorder. We further found weak to moderate factor loadings of OPCRIT ratings on the specific positive, negative and disorganised symptom factor. OPCRIT ratings of manic and depressive symptoms were moderately to strongly associated with the underlying specific symptom dimensions. These findings were first probed in a sensitivity analysis to examine replicability of findings by a bootstrap procedure (online Table DS4). For those parameters for which some relevant bias was detected, absolute values of parameters were estimated to be even larger than the respective point estimate.

\section{Diagnostic utility of general and specific psychosis dimensions}

All latent factor scores were strongly and positively associated with weighted OPCRIT sum scores for general and specific psychosis dimensions (Table 2). This indicated that higher weighted sum scores on a particular dimension can be interpreted as representations of higher latent factor scores.

Symptom profiles for, and findings on differences in, general and specific psychosis dimensions by diagnostic categories are shown in Fig. 1 (see online Fig. DS2 for a colour version and a more detailed description of Fig. 1) and Table 2 respectively.

Scores on the general psychosis $\left(R^{2}=0.81\right)$, specific positive $\left(R^{2}=0.04\right)$, negative $\left(R^{2}=0.02\right)$ and disorganised $\left(R^{2}=0.05\right)$ symptom dimensions were higher for categorical diagnoses of schizophrenia and schizoaffective disorder compared with bipolar disorder. On the manic symptom dimension, scores were lower for schizophrenia than for bipolar disorder $\left(R^{2}=0.20\right)$. Compared with bipolar disorder, scores on the depressive symptom dimension were higher for schizoaffective disorder and lower for schizophrenia $\left(R^{2}=0.18\right)$.

We next examined the utility of general and specific psychosis dimensions for predicting categorical diagnoses using multinomial ROC analysis. Findings on classifying patients into diagnostic categories based on general psychosis dimension compared with classifying patients by chance are shown in online Fig. DS3. Factor scores of the general psychosis dimension yielded a higher proportion of correctly classified patients than each of the specific symptom dimensions alone (Fig. DS3(a)). However, the proportion of patients correctly classified into diagnostic categories based on factor scores of both general and specific psychosis dimensions was markedly higher (95\% CI 0.69-0.79) than the proportions based on the general psychosis dimension only (95\% CI $0.45-0.63$ ), all specific psychosis dimensions combined (95\% CI 0.46-0.57), and what would be expected by chance (95\% CI 0.24-0.35) (Fig. DS3(b)). Online Table DS5 shows corresponding findings from the multinomial regression model with the highest classification accuracy including both general and specific psychosis dimensions for predicting categorical diagnoses.

\section{General and specific psychosis dimensions by clinical variables}

Finally, we examined general and specific psychosis dimensions in relation to clinical variables. Findings on factors scores of general and specific psychosis dimensions by age at onset, mode of onset, premorbid work and social adjustment are shown in Table 2. Scores on the general psychosis dimension were significantly higher for patients with a later age at onset, gradual and insidious (v. acute) mode of onset, poor premorbid work and social adjustment. Table 2 further shows that, compared with patients with an acute onset, those with an insidious onset had lower scores on the positive symptom dimension and higher scores on the negative and depressive symptom dimension. Higher scores on the positive, negative and disorganised symptom dimension and lower scores on the manic symptom dimension were found in patients with poor premorbid work adjustment. Patients with poor premorbid social adjustment also scored higher on the 


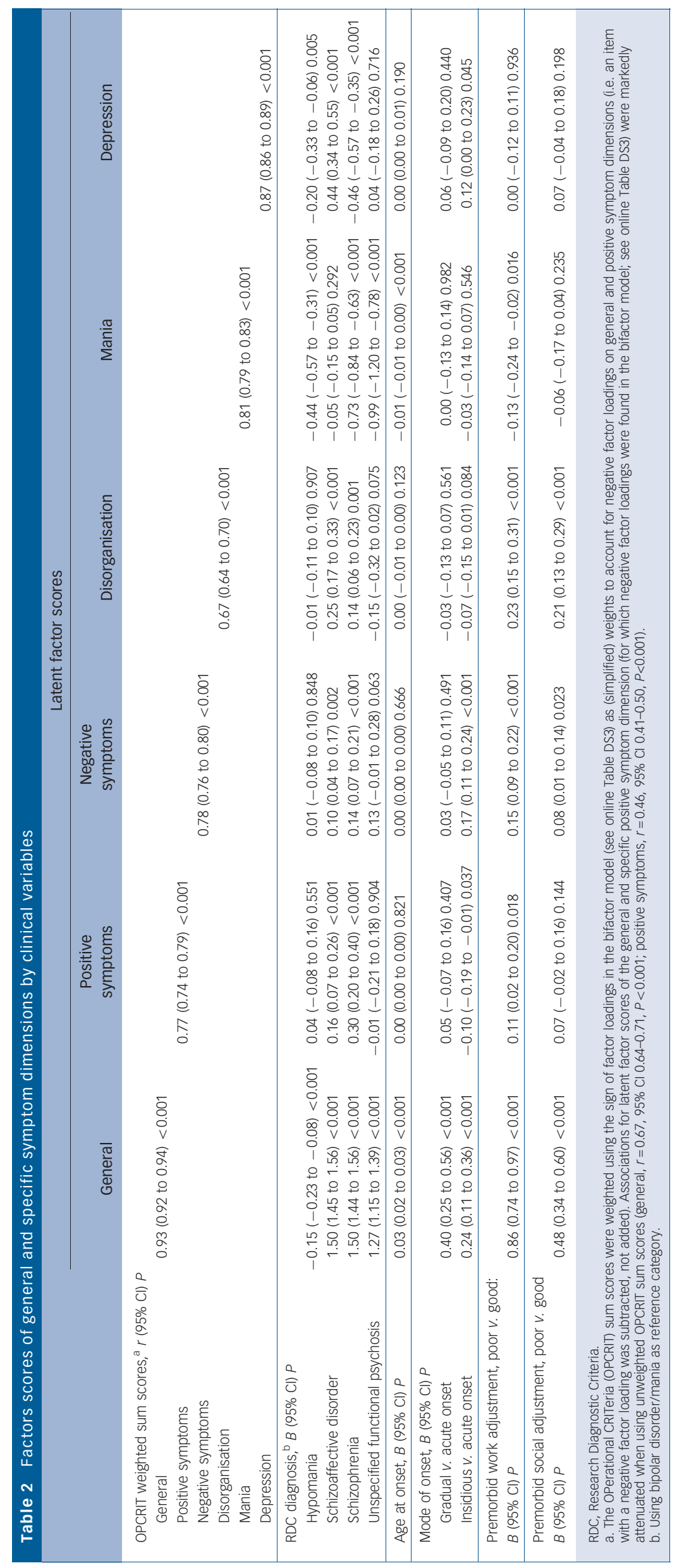




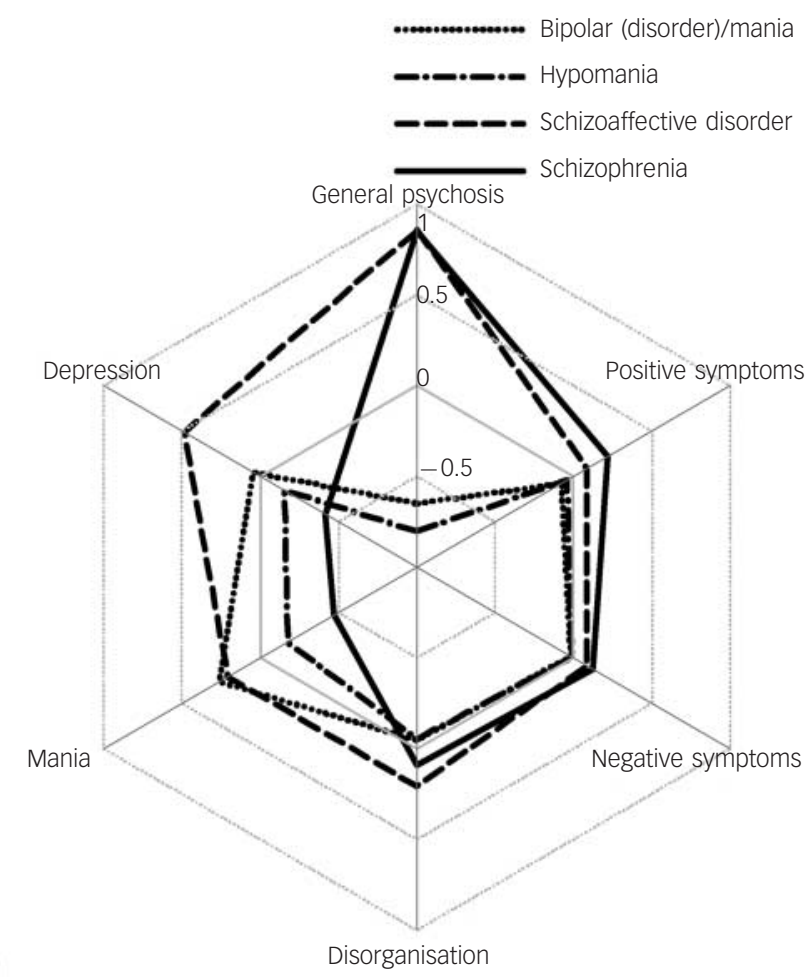

Fig. 1 Symptom profiles for general and specific psychosis dimensions by diagnosis (see online Fig. DS2 for a colour version of this figure).

Symptom profiles are the mean factor scores for one general psychosis dimension and five specific psychosis dimensions (positive symptoms, negative symptoms, disorganisation, mania, depression) by diagnostic categories (schizophrenia, schizoaffective disorder, hypomania, and bipolar disorder/mania). Factor scores are standardised with a mean of 0 and s.d. $=1$.

negative and disorganised symptom dimension than those with good premorbid social adjustment.

\section{Discussion}

\section{Principal findings}

This study is the first to provide evidence for a general psychosis dimension underlying affective and non-affective psychotic symptoms that holds across schizophrenia spectrum and bipolar disorders. Further, there was evidence to suggest formation of specific psychosis dimensions of positive symptoms, negative symptoms, disorganisation, mania and depression is justified. Symptom profiles revealed that general and specific psychosis dimensions discriminated well between, and were consistent with the typical clinical picture of, categorical diagnoses of psychotic disorders. We also found strong evidence on the diagnostic utility when using both general and specific psychosis dimensions for predicting categorical diagnoses. Finally, there was evidence that general and specific psychosis dimensions were differentially associated with age at onset, mode of onset, premorbid work and social adjustment.

\section{Methodological considerations}

We investigated the dimensionality of psychosis in a pooled sample of patients with schizophrenia, schizoaffective disorder, bipolar disorder, hypomania and unspecified functional psychosis. Although this sample allowed for multidimensional item-response modelling of psychotic symptom ratings using a clinical measure directly linked to existing diagnostic classification systems, we were able to include only three OPCRIT items with sufficient prevalence $(>10 \%)$ on negative symptoms, resulting in reduced coverage of this domain, which likely accounted for the limited predictive value of this dimension for categorical diagnoses. Further, given these restrictions on prevalence, the pooled sample size did not provide sufficient power for cross-validation of findings. However, single cross-validations are known to make inefficient use of the data. ${ }^{32}$ Therefore, we used a bootstrap procedure, ${ }^{33}$ providing good evidence on the replicability of findings (Table DS4). Differences in inclusion criteria, OPCRIT rating procedure (online supplement DS1) and prevalence of symptoms (Table DS3) across the two studies, which we purposefully combined in order to achieve sufficient spread in the distribution of non-affective and affective psychotic symptoms, may have led to an artificial increase in variance of both manifest symptoms and latent variables. ${ }^{34}$ Even though difficult to disentangle in this study, we would argue that, given OPCRIT is a well-validated tool purposefully designed to allow flexibility in

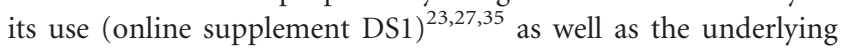
commonalities in phenomenology, aetiology and severity of schizophrenia spectrum and bipolar disorder, such an increase in variance was unlikely to be artificial and instead allowed us to cover a broad range of psychotic disorders. However, ultimately, further investigations that purposefully sample across diagnoses with the same diagnostic assessment methodology are warranted to more fully elucidate this question. ${ }^{3}$ Finally, the current study did not include patients with other relevant non-psychotic disorders, which would have helped to disentangle overlap with, or independence from, other important spectra of mental disorder. ${ }^{4,9,34}$ Multidimensional item-response modelling allowed us to advance on previous research investigating the dimensionality of psychosis by identifying latent dimensions as determinants of symptoms. This approach is now widely considered preferable (for example over principal component analysis), let alone, ignoring the dimensional structure and factorial validity of symptom measures altogether, as common causes and liabilities plausibly lead to symptoms, and not vice versa. ${ }^{34}$ It is also noteworthy that the bifactor model with one general and five specific factors consistently provided a better model fit even when compared with models requiring estimation of the same number of free parameters and, therefore, of identical parsimony. $^{22}$

\section{Comparisons with previous research}

Recent years have seen calls for research cutting across boundaries of diagnostic categories, in order to strengthen the evidence base for including dimensional approaches in updated versions of DSM-5., ${ }^{3,4}$ Our finding of a general psychosis dimension provides evidence, at the clinical symptom level, that cuts across boundaries of the Kraepelinian dichotomy and suggests that schizophrenia and bipolar disorder lie on a transdiagnostic psychosis spectrum with overlapping non-affective and affective symptoms. When directly compared with a model with two distinct factors for non-affective and affective psychosis (a model of identical parsimony), ${ }^{22}$ a superior fit was evident for the bifactor model including one general psychosis factor (and five specific symptom factors). This extends our previous finding of a general psychosis dimension ${ }^{21}$ in schizophrenia spectrum disorder to individuals with bipolar disorder. This dimension resembles, to a degree, the thought disorder factor reported by previous studies, ${ }^{7,36}$ however, the thought disorder factor also included other disorders such as schizotypal and schizoid personality disorders or obsessive-compulsive disorder., ${ }^{7,37}$ Consistent with numerous previous studies, ${ }^{20,21}$ there was also 
evidence that, in addition to this dimension, psychotic symptom ratings are best accounted for by five specific dimensions of positive symptoms, negative symptoms, disorganisation, mania and depression.

In recent years, some researchers have proposed combining dimensional and categorical approaches in the classification of mental disorders, ${ }^{4,20}$ such as ICD- $11^{5}$ and (updated versions of) the DSM-5. ${ }^{2}$ However, to date, there has only been very limited evidence on the diagnostic utility of dimensional representations of psychosis for classifying patients into categorical diagnoses. Overall, strong diagnostic utility of the general and specific psychosis dimensions for allocating patients to diagnoses was demonstrated with the OPCRIT system, a clinical symptom measure that can be used to implement scoring algorithms in both research and routine care. ${ }^{23,27}$ Symptom profiles showed that the general psychosis dimension enabled individuals to be placed on the mania (bipolar disorder/hypomania) $v$. schizophrenia (schizoaffective disorder/schizophrenia) end of the psychosis spectrum. For the specific psychosis dimensions, symptom profiles were consistent with operational definitions of current classification systems $s^{2,37}$ and remarkably similar to those hypothesised for typical patients. ${ }^{20}$ Based on these findings, specific scoring rules can be defined and implemented that allow more accurate classification of patients into these diagnoses. Our findings on symptom profiles may provide a basis for such an approach (online Fig. DS4): first, quantitative scores on the general psychosis dimension may be used to determine whether to place patients on the mania or schizophrenia end of the psychosis spectrum; in a second step, based on the profiles for specific symptom dimensions, patients may be classified into specific diagnoses.

Findings on differential associations between clinical variables and general and specific psychosis dimensions echoed previous reports. ${ }^{7,20,21,38}$ A later, more insidious mode of onset and a poorer premorbid adjustment were associated with the nonaffective end of the psychosis spectrum, whereas an earlier, more acute mode of onset and a better premorbid adjustment related to the affective end of the psychosis spectrum (Fig. DS4). A similar pattern was also evident for the relationship between these variables and the specific symptom dimensions. There is growing evidence that genetic variation and environmental exposures are shared across diagnostic categories. ${ }^{11,12}$ Using the OPCRIT system to derive RDC diagnosis in a twin study, Cardno et al ${ }^{10}$ found evidence of both common and syndrome-specific genetic contributions to psychosis liability. Given also recent calls for identifying cross-cutting dimensions, ${ }^{3,4}$ it is intriguing to speculate whether the general schizophrenia-bipolar disorder psychosis dimension that we have identified here might be strongly linked to shared genetic and environmental risks, ${ }^{9-19,38-40}$ whereas the specific psychosis dimensions are associated with non-shared risks. Although a few studies have investigated intermediate and clinical phenotypes across several different psychotic disorders, ${ }^{41}$ to date, no study that we are aware of has identified and validly measured a transdiagnostic, clinical phenotype of general psychosis underlying affective and non-affective psychotic symptoms in schizophrenia and bipolar disorders. However, ultimately, such a measure, as provided here, is required if we are to move from intermediate to clinical phenotypes and study these in relation to course and outcome of psychosis.

\section{Implications}

Our findings suggest that schizophrenia spectrum and bipolar disorders lie on a transdiagnostic psychosis spectrum with overlapping affective and non-affective psychotic symptoms.
Coupled with strong evidence on diagnostic utility, this finding should inform inclusion of dimensional approaches into (updated versions of) the DSM and may substantially enhance classification accuracy of current diagnostic classification systems. Our findings also provide a directly measurable clinical phenotype for cross-disorder investigations into genetic and environmental factors of psychosis. These are now required to identify shared genetic and environmental contributions to this phenotype (as well as non-shared factors of specific psychosis dimensions) and to disentangle potential overlap with, or independence from, other important spectra of mental disorder.

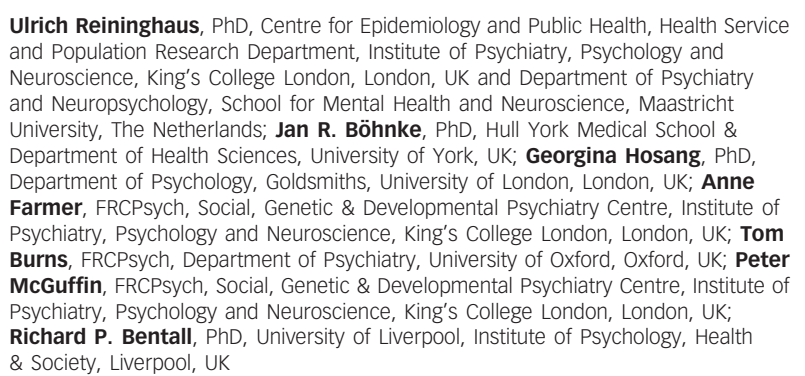

Correspondence: Ulrich Reininghaus, Department of Psychiatry and Neuropsychology, School for Mental Health and Neuroscience, Faculty of Health, Medicine and Life Sciences, Maastricht University, PO Box 616 (VIJV1), 6200 MD Maastricht, The Netherlands. Email: u.reininghaus@maastrichtuniversity.nl)

First received 7 Apr 2015, final revision 26 Aug 2015, accepted 19 Sep 2015

\section{Funding}

U.R. is supported by a Postdoctoral Research Fellowship of the UK National Institute of Health Research (grant no. NIHR-PDF-201104065) and a Veni grant from the Netherlands Organisation for Scientific Research (grant no. 451-13-022). The report is independent research and the views expressed in this publication are those of the authors and not necessarily those of the National Health Service, the National Institute for Health Research or the Department of Health.

\section{References}

1 Kraepelin E. Psychiatry: A Textbook for Students and Physicians (5th edn) [In German]. Verlag von Johann Ambrosius Barth, 1896.

2 American Psychiatric Association. Diagnostic and Statistical Manual of Mental Disorders (5th edn) (DSM-5). APA, 2013.

3 Insel TR. The NIMH Research Domain Criteria (RDoC) project: precision medicine for psychiatry. Am J Psychiatry 2014; 171: 395-7.

4 Adam D. Mental health: on the spectrum. Nature 2013; 496: 416-8.

5 First MB, Reed GM, Hyman SE, Saxena S. The development of the ICD-11 Clinical Descriptions and Diagnostic Guidelines for Mental and Behavioural Disorders. World Psychiatry 2015; 14: 82-90.

6 Bebbington P. Categories, continua and the growth of psychiatric knowledge. Soc Psychiatry Psychiatr Epidemiol 2015; 50: 507-10.

7 Caspi AHR, Belsky DW, Goldman-Mellor SJ, Harrington H, Israel S, Meier MH, et al. The p factor: one general psychopathology factor in the structure of psychiatric disorders? Clin Psychol Sci 2014; 2: 119-37.

8 Carpenter WT, Bustillo JR, Thaker GK, van Os J, Krueger RF, Green MJ. The psychoses: cluster 3 of the proposed meta-structure for DSM-V and ICD-11. Psychol Med 2009; 39: 2025-42.

9 Craddock N, Owen MJ. The Kraepelinian dichotomy - going, going . . . but still not gone. Br J Psychiatry 2010; 196: 92-5.

10 Cardno AG, Rijsdijk FV, Sham PC, Murray RM, McGuffin P. A twin study of genetic relationships between psychotic symptoms. Am J Psychiatry 2002; 159: 539-45.

11 Cross-Disorder Group of the Psychiatric Genomics Consortium. Genetic relationship between five psychiatric disorders estimated from genome-wide SNPS. Nat Gen 2013; 45: 984-94.

12 Lichtenstein $\mathrm{P}$, Yip BH, Bjork C, Pawitan Y, Cannon TD, Sullivan PF, et al. Common genetic determinants of schizophrenia and bipolar disorder in Swedish families: a population-based study. Lancet 2009; 373: 234-9. 
13 Brown AS, van Os J, Driessens C, Hoek HW, Susser ES. Further evidence of relation between prenatal famine and major affective disorder. Am J Psychiatry 2000; 157: 190-5.

14 Clarke MC, Harley M, Cannon M. The role of obstetric events in schizophrenia. Schizophr Bull 2006; 32: 3-8.

15 Matheson SL, Shepherd AM, Pinchbeck RM, Laurens KR, Carr VJ. Childhood adversity in schizophrenia: a systematic meta-analysis. Psychol Med 2013; 43: 225-38.

16 Henquet C, Krabbendam L, de Graaf R, ten Have M, van Os J. Cannabis use and expression of mania in the general population. $J$ Affect Dis 2006; 95 $103-10$

17 Henquet C, Murray R, Linszen D, van Os J. The environment and schizophrenia: the role of cannabis use. Schizophr Bull 2005; 31: 608-12.

18 Heinz A, Deserno L, Reininghaus U. Urbanicity, social adversity and psychosis. World Psychiatry 2013; 12: 187-97.

19 Kirkbride JB, Errazuriz A, Croudace TJ, Morgan C, Jackson D, Boydell J, et al Incidence of schizophrenia and other psychoses in England, 1950-2009: a systematic review and meta-analyses. PLoS One 2012; 7: e31660.

20 van OS J, Kapur S. Schizophrenia. Lancet 2009; 374: 635-45.

21 Reininghaus $U$, Priebe S, Bentall RP. Testing the psychopathology of psychosis: evidence for a general psychosis dimension. Schizophr Bull 2013; 39: 884-95.

22 Russo M, Levine SZ, Demjaha A, Di Forti M, Bonaccorso S, Fearon $\mathrm{P}$, et al. Association between symptom dimensions and categorical diagnoses of psychosis: a cross-sectional and longitudinal investigation. Schizophr Bull 2014; 40: 111-9.

23 McGuffin P, Farmer A, Harvey I. A polydiagnostic application of operational criteria in studies of psychotic illness. Development and reliability of the OPCRIT system. Arch Gen Psychiatry 1991; 48: 764-70.

24 Burns $\mathrm{T}$, Creed $\mathrm{F}$, Fahy $\mathrm{T}$, Thompson $\mathrm{S}$, Tyrer $\mathrm{P}$, White I. Intensive versus standard case management for severe psychotic illness: a randomised trial. Lancet 1999; 353: 2185-9.

25 Cohen-Woods S, Craig I, Gaysina D, Gray J, Gunasinghe C, Craddock N, et al. The Bipolar Association Case-Control Study (BACCS) and meta-analysis: no association with the 5,10-Methylenetetrahydrofolate reductase gene and bipolar disorder. Am J Med Genet B Neuropsychiatr Genet 2010; 153B 1298-304.

26 Spitzer RL, Endicott J, Robins E. Research diagnostic criteria: rationale and reliability. Arch Gen Psychiatry 1978; 35: 773-82.

27 Rucker J, Newman S, Gray J, Gunasinghe C, Broadbent M, Brittain P, et al. OPCRIT+: an electronic system for psychiatric diagnosis and data collection in clinical and research settings. Br J Psychiatry 2011; 199: 151-5.
28 Chalmers RP. mirt: a multidimensional item response theory package for the R environment. J Stat Software 2012; 48: 1-29.

$29 \mathrm{R}$ Core Team. R: A Language and Environment for Statistical Computing (Version 3.0.1). R Foundation for Statistical Computing, 2013.

30 Kass RW, Wasserman L. A reference Bayesian test for nested hypotheses and its relationship to the Schwartz criterion. J Am Stat Assoc 1995; 90: 928-34.

31 Peterson LE, Coleman MA. Machine learning-based receiver operating characteristic (ROC) curves for crisp and fuzzy classification of DNA microarrays in cancer research. Int J Approx Reason 2008; 47: 17-36.

32 Kohavi R. The power of decision tables. Lect Not Comp Sci 1995; 912: 174-89.

33 Efron BG, Gong G. A leisurely look at the bootstrap, the jackknife, and cross-validation. Am Stat 1983; 37: 36-48.

34 Böhnke JR, Croudace TJ. Factors of psychological distress: clinical value measurement substance, and methodological artefacts. Soc Psychiatry Psychiatr Epidemiol 2015; 50: 515-24.

35 Cardno AG, Jones LA, Murphy KC, Murphy KC, Asherson P, Scott LC, et al. Factor analysis of schizophrenic symptoms using the OPCRIT checklist. Schizophr Res 1996; 22: 233-9.

36 Markon KE. Modeling psychopathology structure: a symptom-level analysis of Axis I and II disorders. Psychol Med 2010; 40: 273-88.

37 World Health Organization. The ICD-10 Classification of Mental and Behavioural Disorders: Clinical Descriptions and Diagnostic Guidelines. WHO, 1992.

38 Reininghaus U, Morgan C, Simpson J, Dazzan P, Morgan K, Doody G, et al. Unemployment, social isolation, achievement-expectation mismatch and psychosis. Findings from the ÆESOP Study. Soc Psychiatry Psychiatr Epidemiol 2008; 43: 743-51.

39 Morgan C, Reininghaus U, Fearon P, Hutchinson G, Morgan K, Dazzan P, et al. Modelling the interplay between childhood and adult adversity in pathways to psychosis: initial evidence from the ÆESOP study. Psychol Med 2014; 44: 407-19.

40 Reininghaus $\mathrm{U}$, Craig T, Fisher $\mathrm{H}$, Hutchinson G, Fearon $\mathrm{P}$, Morgan $\mathrm{K}$, et al. Ethnic identity, perceptions of disadvantage, and psychosis. Findings from the AESOP Study. Schizophr Res 2010; 124: 43-8.

41 Tamminga CA, Ivleva El, Keshavan MS, Pearlson GD, Clementz BA, Witte B, et al. Clinical phenotypes of psychosis in the Bipolar-Schizophrenia Network on Intermediate Phenotypes (B-SNIP). Am J Psychiatry 2013; 170: 1263-74. 